\title{
Links between exercise/nutrition and antioxidants-protective effects on immune/respiratory systems as defense against viral infections
}

\author{
Abdurrahman F Kharbat MBA, Stephen Rossettie MBA, Mimi Zumwalt MD
}

\begin{abstract}
This paper discusses factors involved in COVID-19 pathophysiology, with a focus on nutrition, exercise, enzymatic antioxidant systems, and the interplay between immune tolerance and resistance. Of all the supplements, zinc has the most evidence for effectiveness against viruses. However, these data were based primarily on studies measuring duration of the common cold rather than on COVID-19, and optimal dosing remains unclear. Exercise has been shown to have protective tolerogenic effects against viral infection due to the impact of extracellular superoxide dismutases (EC-SODs). Exercise may have a combination of beneficial and harmful effects on outright resistance to viruses in the short term, but taken as a whole it likely has a net protective effect on the immune system. The evidence is examined through the lens of the open window theory and a thorough investigation of the relationship between EC-SODs and exercise/diet. By better understanding the host-virus relationship, clinicians and researchers alike can collaborate to establish guiding principles regarding the steps that individuals can take to protect against some of the deleterious effects of viral infections. More research in this area is needed to understand the relationships among exercise, nutrition, and viral disease.
\end{abstract}

Keywords: COVID-19, SARS CoV-2, nutrition, zinc, EC-SODs, superoxide dismutase, exercise, enzymatic antioxidant/immune systems

\section{INTRODUCTION}

With emergence of the recent SARS-CoV-2 or COVID-19 pandemic, the world has arrived at a previously uncharted health related territory. With the entire human race seemingly taking a moment to recalibrate, this article aims to explain the links between this modern plague, COVID-19, and several determinants of human health, ranging from exercise to nutrition and antioxidants. Viruses are considered opportunistic pathogens that take advantage of lapses in immunity to infect a host. Various factors that protect the

Corresponding author: Mimi Zumwalt Contact Information: Mimi.Zumwalt@ttuhsc.edu DOI: $10.12746 /$ swrccc.v8i36.751 immune and respiratory systems against similar viral infections will be explored.

In this literature review, an attempt is made to answer a number of important questions regarding the protective means that individuals can use to minimize the risk of severe viral infection by COVID-19. The following factors will be addressed:

- Explore the host-virus relationship to explain the pathology of viral infections;

- Investigate the roles of nutrients and antioxidants (dietary and enzymatic) in the development and maintenance of a robust and healthy immune system;

- Characterize the efficacy of exercise as a protective agent against immune dysregulation and tissue damage resulting in atrophy and oxidative stress. 


\section{Methods}

To begin consolidating literature sources, PubMed and Google Scholar were used to search for studies in peer-reviewed journals with keywords relevant to this literature review: COVID-19 viral infection, antioxidants, nutrition, exercise, and immune function. PubMed and Google Scholar were used to search for peer-reviewed scientific studies exploring the interaction among antioxidants, nutrition, exercise, and their effects on immune function and protective effects against viral infections. Although no one source had every element of the following criteria, these criteria were helpful in establishing the integrity and relevance of sources:

1. The source explained relevant information about a subtopic of the proposed field of study.

2. The source provided a previously unexplored perspective on an established policy or intervention.

3. The source focused on elements within the study of protective factors against viral infection and/or immunological function as related to exercise, antioxidants, and nutrition.

4. The source is published in a peer-reviewed journal, by a healthcare agency, or by a reputable nongovernmental organization.

\section{VIROLOGY}

Humans coexist asymptomatically with viruses, both inside and on the body. Studies have found that some humans can produce upward of one trillion $\left(10^{12}\right)$ new viral particles per day. Deleterious effects of viruses usually do not occur unless the virus is exotic, meaning that it naturally occurs asymptomatically in animals, then subsequently transmits infection to humans and damages infected tissue, possibly when humans and animals live closer and closer together over time. For example, HIV, Ebola, and Zika are non-human primate viruses, and Nipah virus is a bat-derived virus, all of which can present as damaging infections in humans. ${ }^{1}$

There are many factors affecting the total disease burden stemming from viruses. The tissue damaging effects of viruses are heavily dependent on age, with young children and the elderly having more serious infections. Moreover, viruses such as the Zika virus can cause congenital defects if pregnant women become infected and the virus is transferred to the fetus during gestation. Dose and route of transmission are also significant considerations. For example, the vaginal epithelium is less susceptible than the anal epithelium for HIV. In addition, host genetics and microbiome are other important factors that will influence the tissue damaging effects of viruses. Moreover, there is a virome in humans, consisting of the other viruses harbored in the body. This could also be significant in the pathogenicity of viral infections. Prior infections and concomitant infections are additional factors that could affect the pathogenesis of viral infections. ${ }^{1}$

While the immune system is a source of resistance to disease, it also has an important role in preventing potential pathogens from having negative effects on health in general. To separate these roles, the ability to limit pathogen burden has been defined as resistance, whereas the ability to limit the health impact of a given pathogen burden has been defined as tolerance. ${ }^{2,3} \mathrm{An}$ example of a tolerance mechanism in the immune system is the elimination of self-reactive T-cell receptors and antibodies. Tolerance mechanisms also include inflammation reduction or checkpoints that reduce autoimmunity by selecting for immune-activating molecules, such as antimicrobial peptides or toll-like receptors, that are less reactive to self than to pathogens. ${ }^{3}$

Some immune factors can have a trade-off between resistance and tolerance. Tumor necrosis factor (TNF), for example, is an immune cell signaling molecule that is important for proper resistance, but it can simultaneously decrease tolerance and lead to host damage due to the increase in generalized, nonspecific inflammation. While much of this article will examine factors of immunology by considering resistance and tolerance, these factors are strongly related and thus immunology may be discussed in some cases without separating these two concepts. ${ }^{3}$

\section{NUTRITION}

Under certain conditions, oxygen can react and "mutate" into a number of chemical compounds referred to as free radicals or reactive oxygen species (ROS), 
which can have deleterious effects on cells and tissues in the body. ${ }^{4}$ Increased ROS are strongly associated with asthma, COPD, and other respiratory diseases that may lead to a worse prognosis in COVID-19 patients, but it is unclear whether ROS are causative factors or the consequence of such diseases. There is strong evidence that supplemental antioxidants can help in people with deficient nutritional antioxidants, but existing data do not show consistent benefits in people with adequate (normal levels) antioxidant intake. Furthermore, antioxidant supplements can act as pro-oxidants, or ROS inducers, when consumed above the recommendation doses. Therefore, supplemental antioxidants may be indicated in specific scenarios, but there is limited evidence regarding benefits on respiratory health in people without a specific antioxidant deficiency. ${ }^{5}$

Zinc stands out as a supplement that may have practical benefits against viral infections. In a meta-analysis of seven randomized, double-blind, and placebo-controlled trials, oral lozenges of zinc reduced the duration of the common cold by $33 \%$ compared to the control group. ${ }^{6}$ The proposed mechanism, explained in another study, is largely due to zinc's antioxidant and anti-inflammatory "tolerogenic" effects on the immune system. The same study showed evidence that probiotics may have resistive and tolerogenic effects against upper respiratory tract viral infections due to increased mucosal immunity, but the evidence has been rated as low quality. ${ }^{2}$ Optimal dosing of zinc lozenges or probiotics is still not clear; five of the trials in the meta-analysis used zinc doses of 80-92 mg/day. ${ }^{6}$

Nutrition can also affect viral diseases, which in turn are compounded by aging. The relationship between malnutrition and aging is bidirectional-an increase in either factor increases the other. Regulation of energy intake is significantly decreased in older populations (human and animal). This can be explained by several factors, including decreased basal metabolic rate or increased cholecystokinin in the elderly, especially after high fat meals. The mechanisms of immune compromise associated with malnutrition are not fully understood. One probable explanation lies in a lack of building blocks (amino acids) and energy (ATP or equivalents) leading to a deficiency in immune infrastructure. However, these conditions do not complete the picture because such insufficiency is not uniformly distributed in patients with marasmus, a state of severe undernourishment. Strangely, these patients have adequate or even increased levels of certain immune parameters, such as plasma lgA, acute-phase proteins, leukocytes, and the production of Th2 cytokines, thus the "insufficient building blocks" theory must be partially flawed. Another explanation attributes the connection between malnutrition and immune suppression to problems with growth hormone or thymic function. This theory could explain why zinc lozenges may be beneficial to immunity. Zinc deficiency causes thymic atrophy, and acute phase responses lower plasma zinc levels. Therefore, zinc levels may contribute to the immune deficiency of both malnutrition and acute phase responses. ${ }^{7}$

Obesity, another form of malnutrition, has significant effects on respiratory illness. This is due largely to the alteration in adipokines, especially adiponectin and leptin. As such, a foundational understanding of the importance of nutrition is essential to ensure that the appropriate nutrients are consumed by individuals, both for the purposes of excess weight reduction and appropriate nutrient and vitamins/elemental intake. Weight loss interventions can significantly improve outcomes in respiratory disease. Moreover, foods with essential nutrients have an integral role in providing the human body with the sustenance needed to support a healthy and robust immune system, to promote immune responses, and to reduce airway inflammation in respiratory illness. ${ }^{8}$ See Figure 1.

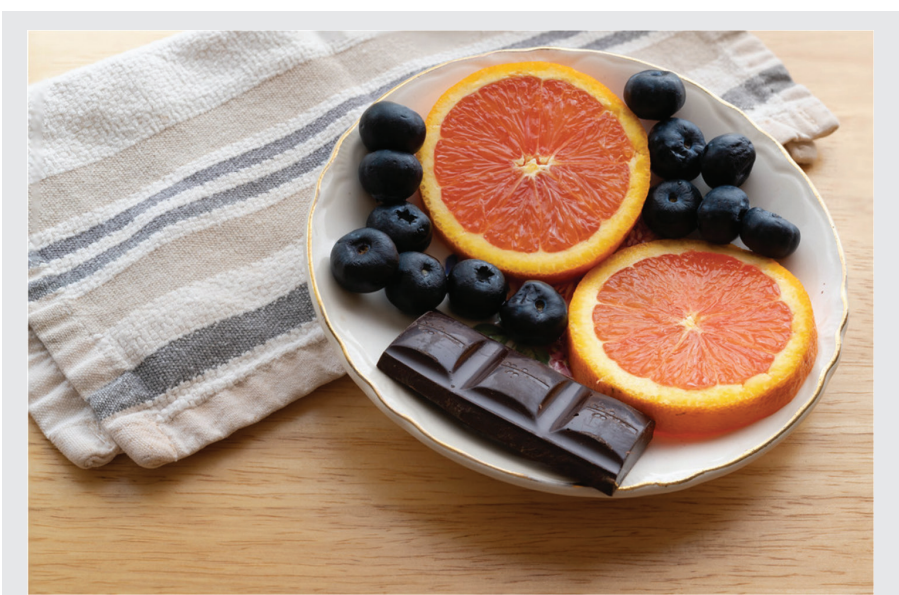

Figure 1. Antioxidant Food Sources-Image obtained from Creative Commons. 
Table 1. Sources of Antioxidants

\begin{tabular}{|l|l|}
\hline Antioxidants & Sources \\
\hline Ascorbic acid & $\begin{array}{l}\text { Citrus fruits, tomatoes, vegetables, } \\
\text { brown rice, pink and red wine, mango, } \\
\text { papaya, sweet potato, watermelon }\end{array}$ \\
\hline Catechins & $\begin{array}{l}\text { Apples, beans, berries, wine, tea, } \\
\text { fruits, chocolate }\end{array}$ \\
\hline Anthocyanins & $\begin{array}{l}\text { Beets, berries, eggplant, grape fruits, } \\
\text { pink and red wine }\end{array}$ \\
\hline Beta-carotene & $\begin{array}{l}\text { Vegetables, tomatoes, carrots, sweet } \\
\text { potatoes, apricots, papayas, squash, } \\
\text { acorns, peaches, bell peppers, broccoli, } \\
\text { mango }\end{array}$ \\
\hline Polyphenols & $\begin{array}{l}\text { Tea, fruits, vegetables, red cabbage, } \\
\text { blue and black berries }\end{array}$ \\
\hline Lycopene & $\begin{array}{l}\text { Tomatoes, papaya, watermelon, guava, } \\
\text { pink and red grapes }\end{array}$ \\
\hline Tocopherol & $\begin{array}{l}\text { Cereals, broccoli, Brussels sprouts, } \\
\text { beans, cauliflowers, cooking oils } \\
\text { (olive, sunflower, safflower), almonds, } \\
\text { hazelnuts, whole grains, wheat germ }\end{array}$ \\
\hline Lutein & Corn, egg \\
\hline Selenium & $\begin{array}{l}\text { Carrots, chicken, garlic, onions, } \\
\text { oatmeal, salmon, tuna, seafood, whole } \\
\text { grains, wheat germ }\end{array}$ \\
\hline
\end{tabular}

Data from Mbah et al. ${ }^{11}$

Lycopene is an antioxidant that has been shown to suppress neutrophilic airway inflammation. Vitamin $\mathrm{C}$ has been demonstrated in vitro to suppress NF- $\mathrm{KB}$, TNF- $\alpha$, and IL-8, but it is unclear whether vitamin C supplementation is beneficial in vivo. Similarly, supplemental vitamin $E$ and $D$ have also led to inconsistent results, because it is challenging to control for a certain nutrient when people have diets composed of many interacting nutrients. Still, it is important to note that adequate diets are important since undernutrition worsens COPD and other respiratory diseases. ${ }^{8}$ See Table 1.

While studies on nutritional supplements are important in the search for optimal health, supplementation needs to be considered within the context of an individual's diet and any known genetics or nutritional deficiencies. For respiratory diseases, the Mediterranean diet, which consists of healthy natural fats and proteins, appears to be the most protective. Studies of individual nutrients have too many limitations to be conclusive, but evidence does show that an increase in fruit and vegetable intake is associated with improved respiratory function/outcomes. ${ }^{8}$ Last, it is especially important that athletes consume a well-balanced diet that is sufficient to meet requirements of energy, protein, and micronutrients because intense exercise and poor nutrition can act synergistically to stress the body and consequently weaken the immune system. However, with proper nutrition, exercising may help to support the immune system in many ways, including partitioning ROS with enzymes called superoxide dismutases (SODs) ${ }^{9}$

\section{SUPEROXIDE DISMUTASE AND EXERCISE}

Superoxide dismutases (SODs) are postulated to have existed for billions of years and may be some of the oldest enzymes crucial for life forms. They have adapted over the years to fit into contemporary biology and serve many essential-to-life roles in physiological processes. Various isoforms exist in subcellular locations that have specific actions. ${ }^{10}$ All of these help to neutralize, or "dismutate", reactive oxygen species (ROS) to decrease potential oxidative damage to cells and tissues (specifically to endothelial surfaces). By analyzing the immune system's defenses against ROS and the relationship between exercise and one of the SOD subtypes, it was observed that exercise, by upregulating these enzymes, may help to protect against viruses, such as SARS CoV-2. ${ }^{4}$

Superoxide dismutases serve an important function in eliminating superoxides from tissues, which have the potential to cause oxidative damage. Superoxide dismutases, in conjunction with catalase (CAT) and glutathione peroxidase (GPX), catalyze the reduction of superoxide species into hydrogen peroxide and ultimately back into water. Despite this defense mechanism, oxidative damage can still occur in the body if these enzymes become saturated. ${ }^{4}$

The oxidative damage often involves the oxidation of DNA/RNA, proteins, lipids, and other 
Table 2. Superoxide Dismutase Isoenzymes

\begin{tabular}{|l|l|l|l|}
\hline Isoenzyme & Name & Characteristics & Cellular Localization \\
\hline SOD1 & CuZnSOD & $\begin{array}{l}\text { Copper and zinc-containing } \\
\text { homodimer }\end{array}$ & Cytoplasm \\
\hline SOD2 & MnSOD & $\begin{array}{l}\text { Tetrameric manganese-containing } \\
\text { enzyme }\end{array}$ & Matrix of mitochondria \\
\hline SOD3 & EC-SOD & $\begin{array}{l}\text { Copper and zinc-containing } \\
\text { tetrameric enzyme }\end{array}$ & $\begin{array}{l}\text { Secreted by producing cells } \\
\text { into extracellular space }\end{array}$ \\
\hline
\end{tabular}

Data from Yan et al. ${ }^{4}$

macromolecules and has been implicated in multiple pathological disease processes. ${ }^{11}$ Of the three SOD isoenzymes, most pertinent to this review is SOD3, or extracellular superoxide dismutase (EC-SOD), which is a copper and zinc-containing tetrameric enzyme secreted from cells that contain this isoenzyme into the surrounding extracellular space. This isoenzyme has significance to the relationship between exercise and immunology because endurance exercise induces EC-SODs in skeletal muscle cells and allows for their dissemination in other peripheral structures, leading to a systemic effect of reducing free radicals and reducing oxidative stress on endothelial surfaces. ${ }^{4}$ See Table 2.

In addition to being stimulated by exercise, EC-SOD is unique due to its extracellular existence. While most SODs function intracellularly, the extracellular presence of EC-SOD allows the dismutation of superoxides in various organs, most notably in the lungs and kidneys. Detoxification of vascular superoxides by EC-SOD preserves the bioavailability of nitric oxide (NO) by decreasing oxidative damage of endothelial surfaces and maintaining normal vasoreactivity. The functional importance of EC-SODs can be best exemplified by observing diseases that involve loss-of-function of EC-SODs, which are characterized by increased risk of oxidative stress lending to poor prognosis, such as in acute pulmonary injury. Ultimately, EC-SODs have emerged as a leading plausible therapy for disease processes that are characterized by increased risk of oxidative stress and tissue damage, such as coronavirus and influenza viral infections. ${ }^{4}$
Coronaviruses and influenza virus can cause lethal lung injuries and death from Acute Respiratory Distress Syndrome (ARDS) by evoking a "cytokine storm" that leads to increased oxidative stress and free radicals, among other factors. The hallmark of ARDS is characterized by severe hypoxemia and usually involves uncontrolled inflammation, oxidative injury, and damage to the alveolar-capillary barrier. As such, EC-SODs and other chemicals that reduce harmful free radicals may have an important role in the treatment of ARDS-inducing viruses. ${ }^{4,12}$

Endurance exercise has been shown to induce EC-SODs in skeletal muscle cells allowing for their dissemination to other peripheral structures and to lead to a systemic effect of reducing oxidative stress by neutralizing ROS and decreasing damage of endothelial surfaces. ${ }^{4}$ In a 2018 study, aerobic exercise resulted in increased superoxide dismutase SOD $(p<0.01)$ and decreased superoxide anion accumulation in the lungs $(p<0.01) .^{13}$ In fact, it has been conclusively demonstrated that regardless of intensity, volume, type of exercise, and sample population, antioxidant activity increases and pro-oxidant indicators decrease following exercise sessions. This phenomenon is due to enhanced antioxidant defenses in contractile tissues, such as the increased capacity of antioxidants, such as EC-SOD, in slow-twitch muscle fibers, which is needed to neutralize the increased generation of ROS due to their oxidative nature, as compared to fast-twitch muscle fibers, which use glycolytic metabolism. This increased capacity is achieved through promotion of EC-SOD gene transcription and protein expression in skeletal muscles. ${ }^{4}$ 
However, antioxidants, such as SODs, are not the only factors that reduce tissue damage caused by ARDS. The same 2018 study showed that aerobic exercise has anti-inflammatory effects through a separate mechanism, via the cytokine IL-10. These investigators found that aerobic exercise inhibited LPS-induced lung inflammation by attenuating inflammatory cytokines and oxidative stress markers in mice and human cell culture via enhanced IL-10 production in addition to SODs. ${ }^{13}$

The benefits of increased EC-SODs as a result of exercise extend to many other pathologic states, including cachexia. Cachexia is a clinical syndrome characterized by weight loss due to muscle and adipose tissue wasting and inflammation, which is seen in multiple chronic diseases. Most notably among these are cancer, diabetes, chronic obstructive pulmonary disease, congestive heart failure, and renal disease, all of which are associated with oxidative stress. Oxidative stress has been demonstrated to critically affect cardiac cachexia, with significantly lower EC-SOD activity and quantity within skeletal muscles in these patients. However, slow-twitch oxidative muscles with large antioxidant capacities are resistant to catabolic muscle wasting and, therefore, are not at risk for the atrophy of cachexia. This further increases understanding of the protective effects of EC-SODs and demonstrates the therapeutic potential of exercise as a protective factor against muscle and adipose tissue degeneration along with inflammation seen in various diseases. The molecular mechanism underlying this protective effect is NO-mediated enhancement of endothelial function, by way of EC-SODs counteracting dysfunction of the endothelium, which allows vasoreactivity and normal endothelial function to persist, maintaining blood flow and perfusion to various tissues, thus diminishing oxidative stress and subsequent catabolism. ${ }^{4}$

Cardiovascular disease is the leading cause of death in Americans, and oxidative stress has been demonstrated to have an active role in the pathophysiology of this disease process. Among the numerous cardiovascular diseases, hypertension (HTN), atherosclerosis, myocardial infarction (MI), and chronic heart failure (CHF) are pathologies with demonstrated oxidative stress components. Since COVID-19 is more likely to severely affect patients with underlying cardiovascular disease, an understanding of the protective role of antioxidants in the pathophysiology of these diseases might demonstrate the efficacy of antioxidants and the induction of EC-SODs through exercise as a possible protective factor in the prevention of severe COVID-19 symptoms. The endothelial surfaces have a role in resistance in arteries, are responsible for altering vascular tone, and therefore regulate blood pressure. Endothelial dysfunction, a consequence of oxidative damage, is a product of impaired endothelium-dependent vasodilation and has an important role in the pathogenesis of HTN. Therefore, EC-SOD induction could protect against HTN, especially in the context of high risk COVID-19 patients. Moreover, atherosclerosis has been demonstrated to be one of the most effectively targeted cardiovascular diseases through exercise, and this is in part due to the induction of EC-SODs, as atherosclerotic vessels have high levels of superoxides in hypercholesterolemic mice and in older humans, suggesting a role of ROS in the pathogenesis of atherosclerosis. Exercise-induced increases in EC-SODs help reduce excess ROS and decrease the oxidative stress placed on stiff blood vessels. Consistent with these findings are the observations that patients with $\mathrm{MI}$ and $\mathrm{CHF}$ are heavily affected by oxidative stress, and the demonstration that exercise-induced EC-SODs plus the antioxidant effects of enzymatic mechanisms in reducing the severity of these pathologies, thus having a protective role against cardiovascular disease. ${ }^{4}$

The above observations/findings could be helpful for understanding the pathogenesis of COVID-19 and the role of antioxidants in the mitigation of endothelial dysfunction due to oxidative stress. There is no evidence on the direct interactions between COVID-19 and EC-SODs yet, but the observations seen between EC-SODs and viruses may indicate that aerobic exercise-through its induction of EC-SODs-could potentially provide some level of protection against the endothelial dysfunction caused by COVID-19. ${ }^{4}$

As for the respiratory system, oxidative stress contributes to the pathologic process in pulmonary 
disease, since EC-SOD is highly expressed in the extracellular matrix of lung tissue, especially at the junctions of airway epithelial cells and the endothelium of bronchial smooth muscles of the lungs. The preponderance of evidence indicates that EC-SOD polymorphisms that result in loss-of-function have a large role in the oxidative stress that affects the lungs in diseases such as acute respiratory distress syndrome (ARDS). Innate immunity has a profound role in the pathogenesis of ARDS, which involves cascades of neutrophils, macrophages, and dendritic cells causing pulmonary damage. Excessive neutrophil activation and accumulation, which is an integral component of ARDS, results in the generation of ROS which then leads to oxidative stress. In an experiment testing the protective nature of EC-SOD induction via exercise, researchers found that enhanced EC-SOD expression in skeletal muscles of mice protected against ARDS, while EC-SOD knock-out mice became increasingly more susceptible to tissue injury. These findings indicate that promotion of EC-SOD expression in skeletal muscle, which can be systemically redirected to the lungs, could be a viable preventative and protective measure in reducing the risk and severity of ARDS. ${ }^{5,11}$

In addition to its cardiovascular and pulmonary benefits, EC-SOD induction has provided some protection against renal disease. Although the link to this protective effect is poorly understood, it has been demonstrated through induction via exercise. Moreover, knock-out EC-SOD mice that received blood transfusions from EC-SOD expressing mice were protected against oxidative stress on renal tissues, indicating the role of systemic EC-SOD in providing a protective effect. This could be explored as a protective mechanism in individuals with an inability to induce EC-SOD via exercise, e.g., severely immunocompromised or bed-ridden patients. ${ }^{4,11}$

Finally, one of the highest contributors of ICU mortality is multiple organ dysfunction syndrome (MODS), in which numerous organs are damaged to a point at which systemic homeostasis cannot be achieved nor maintained. It has been observed that this is one of the contributors of death in many COVID-19 cases. ${ }^{14}$ Emerging evidence indicates that ROS has a role in inducing endothelial cell activation and in the pathogenesis of MODS in humans. In clinical trials, general antioxidants have failed as therapeutic interventions due to the lack of target specificity. However, through parabiosis experiments and intraperitoneal serum transfusions, researchers have demonstrated that EC-SOD delivered protective effects against MODS by preserving lung function, reducing endothelial cell activation, and decreasing endothelial cell adhesion. It is speculated that serum transfusions and other ways of transplanting EC-SODs to COVID-19 patients undergoing oxidative stress could be a mechanism of decreasing the morbidity and mortality of MODS and other instances of tissue/organ failure..$^{4,14}$

\section{Open Window Theory}

Although exercise can reduce damage caused by pathogens and immune-mediated responses, it also may have some immune compromising effects in the short term. Following an exercise session, there is an "open window" of immunosuppression while recovering that, with repeated bouts of exercise without recovery, can leave an individual susceptible to illness. Some studies estimate a $15-70 \%$ decrease in both innate and acquired immunity after exercising. One explanation for this effect is that stress from excessive or prolonged exercise increases glucocorticoids that have anti-inflammatory effects, thus leading to increased susceptibility to disease. ${ }^{2}$

However, this immunosuppression is elusive and has only been quantified explicitly in decreased salivary IgAlevels. Moreover, exercise results in increased circulating levels of neutrophil and monocyte counts and decreased lymphocyte count during recovery. ${ }^{15}$ Many of the studies that show increased incidence of URI in elite athletes after competition have significant limitations, and more recent studies have shown that anxiety and travel may have an important modulating roles of exercise/glucocorticoids in the open window theory. ${ }^{2}$

Further studies have shown that even if exercise may lead to a short-term decrease in immune resistance, it may also result in an increase of the immune system tolerance to disease. This is because high intensity exercise creates an anti-inflammatory response 
that could cause a decrease in skeletal muscle damage. Moreover, this anti-inflammatory response has been considered to be mediated through the induction of EC-SODs, which protect against inflammation by reducing oxidative stress. An exercise level of moderate intensity promotes protection against infections caused by intracellular microorganisms, since it guides the immune response to a predominance of Th1 cells. Conversely, high-intensity activities increase the concentrations of anti-inflammatory cytokines (Th2 pattern), presumably to decrease damage in muscular tissue resulting from inflammation, although it still may result in a short term increase in susceptibility to infections. $^{16}$

\section{Discussion}

By better understanding the interplay of the host-virus relationship, clinicians and researchers alike can establish guiding principles regarding the steps that individuals can take to protect against some of the harmful effects of viral infections, especially with COVID-19, which causes system-wide pathology. Tolerance aims to minimize the deleterious effects resulting from incumbent pathogen populations, whereas resistance aims to reduce the pathogen population size within the host. More research is needed to better comprehend how to increase tolerance without effecting a simultaneous decrease in resistance, but understanding how these two factors of immunity interact with each other and with exercise shows that people are likely to see benefits from exercise, especially when combined with adequate nutrition. ${ }^{3}$

Malnutrition increases with age, largely due to decreased hunger drive and an increased sense of satiety, most likely due to declining metabolism. This progressive malnutrition has been characterized by and correlated with diminished muscle function, reduced bone mass, immune dysfunction, anemia, reduced cognitive function, poor wound healing, delayed recovery from surgery, higher hospital readmission rates, and increased morbidity/mortality. In the context of protection against viral infections such as COVID-19, a fundamental protective effect against severe disease is ensuring that older individuals are able to take in the required proper nutrients to maintain a robust immune system and to minimize tissue degeneration and atrophy. Although decreased hunger and increased satiety may contribute to smaller volumes of food intake, an important step that older individuals should take is to ensure that essential vitamins and antioxidants are consumed in their diet. Although supplementation of vitamins/minerals and antioxidants beyond the baseline recommended in a healthy diet have not been demonstrated to add value in the context of protective effects, ensuring that a baseline level of these nutrients are being taken in is essential. As such, a recommendation is made that individuals who do not/cannot obtain these nutrients in their normal diet should incorporate vitamins/ minerals and antioxidants through supplementation, after consultation with their primary care physician. ${ }^{7}$

Finally, it is important that athletes have a wellbalanced diet that is sufficient to meet requirements of energy, protein, and micronutrients since heavy exercise and poor nutrition act synergistically to stress the body and further weaken the immune system. Moreover, it is important to highlight the importance of regular exercise, not only for athletes who aim to stay in shape and are already exercising, but more important for individuals who have a sedentary lifestyle. By combating obesity, strengthening the immune system, and inducing EC-SODs to reduce inflammation and decrease oxidative stress, exercise should be considered one of the best protective factors against viral infections such as COVID-19. ${ }^{9}$

\section{Conclusions}

1. Supplements are recommended in specific scenarios, such as athletes on energy-restricted diets and the elderly exhibiting reduced food intake, but otherwise should be taken carefully as excess vitamins can also have immunosuppressive effects. ${ }^{9}$

2. Athletes should eat a well-balanced diet sufficient to meet their energy requirements in order to maintain proper immunity after exercise. This is because training in a carbohydrate-depleted state will increase circulating stress hormones and change the traffic pattern of immune cells, such as leukocytes and lymphocytes. ${ }^{9}$ 
3. It is very important that people who are already infected with a virus do not return to heavy exercise until the infection has been resolved completely, as this could lead to serious complications, such as myositis, rhabdomyolysis, and myopericarditis. ${ }^{2}$

4. Exercise is a first-line of defense against oxidative stress, through the actions of extracellular superoxide dismutases (EC-SODs), which is released from skeletal muscle to the systemic circulation and tissues by exercising. Therefore, the positive effects of exercise should be pursued by all those who wish to protect against the deleterious effects of viral infections such as COVID-19. ${ }^{4}$

Article citation: Kharbat AF, Rossettie S, Zumwalt M. Links between exercise/nutrition and antioxidantsprotective effects on immune/respiratory systems as defense against viral infections. The Southwest Respiratory and Critical Care Chronicles 2020;8(36): 23-31

From: School of Medicine, Texas Tech University Health Sciences Center, Lubbock, Texas

Submitted: $8 / 10 / 2020$

Accepted: 9/20/2020

Reviewer: Kenneth Nugent MD

Conflicts of interest: none

This work is licensed under a Creative Commons

Attribution-ShareAlike 4.0 International License.

\section{REFERENCES}

1. Sumbria D, Berber E, Rouse BT. Factors affecting the tissue damaging consequences of viral infections. Frontiers Microbiology 2019;10:2314.

2. Walsh NP. Nutrition and athlete immune health: new perspectives on an old paradigm. Sports Medicine 2019 Nov $6: 1-6$.

3. Ayres JS, Schneider DS. Two ways to survive an infection: what resistance and tolerance can teach us about treatments for infectious diseases. Nature Reviews. Immunology 2008 Nov;8(11):889.

4. Yan Z, Spaulding HR. Extracellular superoxide dismutase, a molecular transducer of health benefits of exercise. Redox Biology 2020 Mar 19:101508.

5. Liu Z, Ren Z, Zhang J, et al. Role of ROS and nutritional antioxidants in human diseases. Frontiers Physiology 2018 May 17;9:477.

6. Hemilä H. Zinc lozenges and the common cold: a meta-analysis comparing zinc acetate and zinc gluconate, and the role of zinc dosage. JRSM open 2017 Apr;8(5): 2054270417694291.

7. Alam I, Almajwal AM, Alam W, et al. The immunenutrition interplay in aging-facts and controversies. Nutrition and Healthy Aging 2019 Jan 1;5(2):73-95.

8. Berthon BS, Wood LG. Nutrition and respiratory healthfeature review. Nutrients 2015 Mar;7(3):1618-43.

9. Gleeson M, Nieman DC, Pedersen BK. Exercise, nutrition and immune function. J Sports Sciences 2004 Jan 1; 22(1):115-25.

10. Case AJ. On the origin of superoxide dismutase: an evolutionary perspective of superoxide-mediated redox signaling. Antioxidants 2017 Dec;6(4):82.

11. Mbah CJ, Orabueze I, Okorie NH. Antioxidants properties of natural and synthetic chemical compounds: therapeutic effects on biological system. Acta Scientific Pharmaceutical Sciences 3.6 (2019):28-42.

12. Cheng RZ. Can early and high intravenous dose of vitamin C prevent and treat coronavirus disease 2019 (COVID-19)? Medicine Drug Discovery 2020 Mar;5:100028.

13. Rigonato-Oliveira NC, MacKenzie B, Lacerda Bachi AL, et al. Aerobic exercise inhibits acute lung injury: from mouse to human evidence Exercise reduced lung injury markers in mouse and in cells. Exercise Immunology Review 2018 Jan $1 ; 24$.

14. Zaim S, Chong JH, Sankaranarayanan V, et al. COVID-19 and multi-organ response. Current Problems Cardiology 2020 Apr 28:100618.

15. Peake JM, Neubauer O, Walsh NP, et al. Recovery of the immune system after exercise. J Applied Physiology 2017 May 1;122(5):1077-87.

16. Terra R, Silva SD, Pinto VS, et al. Effect of exercise on the immune system: response, adaptation and cell signaling. Rev Bras Med Esporte 2012 May 1;18(3):208-14. 